\title{
L'APD entre promesses non tenues et nouveaux partenariats
}

Vincent RIBIER

CIRAD, 45 bis avenue de la Belle-Gabrielle, 94736 Nogent-sur-Marne cedex,

France

<vincent.ribier@cirad.fr>

\begin{abstract}
The article refers to the profound transformation of ODA in recent years. It is primarily the observation that the promises made by donor countries are rarely kept, a situation which reveals more of a culture of non-compliance with commitments than economic difficulties forcing donors to delay their commitments. This finding is particularly true for agricultural ODA as evidenced by the low share of disbursed amounts promised when the food crisis of 2008. The article then focuses on the qualitative aspects of aid noting the quest for new forms of partnership expressed in the Paris Declaration. It stresses the difficulty in operationalizing the principles of this Declaration, including the first of them, ownership.
\end{abstract}

Key words: official development assistance, donor countries, agricultural ODA, Paris declaration, new forms of partnership

des modalités de celle-ci, mais aussi du nombre de ses contributeurs. Le comité d'aide au développement (CAD) de l'OCDE ne recense que les montants de I'APD versés par ses pays membres, alors même que de nouveaux acteurs pèsent de plus en plus lourds dans les transferts financiers en direction du Sud pour lesquels il n'existe pas de statistiques fiables: les montants engagés par les bailleurs émergents hors OCDE, par le secteur privé, par les fondations (par exemple la fondation Gates, devenue un acteur important de l'APD agricole), par les ONG, sont peu diffusés (Gabas, 2008).

Il est par ailleurs difficile de distinguer, dans les décaissements réellement effectués, la part qui relève des nouveaux engagements de celle correspondant aux financements déjà programmés antérieurement. Certaines promesses d'aide qui semblent annoncer l'arrivée d'argent frais correspondent en fait à des programmes déjà en cours. II est également difficile de connaître le contenu exact des engagements et leurs modalités d'attribution : s'agit-il d'aide budgétaire ou d'aide projet? S'agit-il de dons ou de prêts? La base d'engagement est-elle annuelle ou pluriannuelle? (Pouilles Duplaix, 2009)

Compte tenu de ces difficultés méthodologiques, l'analyse ci-après se base essentiellement sur les flux d'aide en provenance des pays de I'OCDE, que ce soit sous forme bilatérale ou comme contribution des États aux organismes multilatéraux. Après la baisse des années 1990 (58 milliards de dollars courants en début de période, 52 milliards en fin), I'APD nette des pays de l'OCDE a augmenté au cours de la première moitié des années 2000 pour atteindre 107 milliards en 2005. L'aide s'est tassée depuis. Cette progression en dollars courants est à relativiser si on ramène $\mathrm{I}^{\prime} \mathrm{APD}$ au PIB des pays donateurs. Cette part du PIB consacrée à I'APD est en effet inférieure actuellement à ce qu'elle était au début des années 1990. De $0,33 \%$ en 1990 , ce pourcentage est progressivement tombé jusqu'à 0,22 \% sur la période 1997-2001 avant de remonter en 2005 jusqu'au 0,33\% initial, puis de redescendre à $0,30 \%$ en 2008 (figure 1).

Ces différents éléments chiffrés montrent à quel point les promesses d'engagements financiers n'ont pas été tenues :

- le fameux objectif de 0,7 \% du PIB semble de moins en moins crédible, malgré les promesses réitérées de l'atteindre à une échéance donnée. Le problème est que cette échéance est sans cesse repoussée, ainsi que les échéances intermédiaires censées être une étape vers l'atteinte des $0,7 \%$. Ce constat s'applique à tous les niveaux, celui des pays membres de I'OCDE, celui de l'Union européenne ou celui de nombreux pays ;

- seuls quelques pays dépassent, parfois nettement, l'objectif des 0,7 \% du PIB. II s'agit principalement des pays nordiques, selon les données fournies par l'OCDE pour 2008 : $0,98 \%$ pour la Suède, $0,88 \%$ pour la Norvège, $0,82 \%$ pour le Danemark ou encore $0,80 \%$ pour les Pays-Bas. La contribution de I'Allemagne n'est que de 0,38\%, tandis que celle de la France n'est guère supérieure avec $0,39 \%$. La promesse de Jacques Chirac de monter I'APD de la France à $0,5 \%$ du PIB en 2007 fait d'ores et déjà partie des engagements non tenus;

- la performance globale des pays membres de I'OCDE est tirée vers le bas par les poids lourds États-Unis et Japon, dont la contribution respective n'est que de $0,18 \%$, proche des plus

Il est malaisé d'estimer de manière précise les montants totaux de l'APD, du fait de la diversité 


\section{Quelques promesses des années 2000}

- La conférence de Monterrey, consacrée en mars 2002 au financement du développement, se conclut sur l'adoption d'un consensus qui invite notamment les pays développés à consacrer $0,7 \%$ de leur PIB en faveur de I'APD et à affecter une part de 0,15 à 0,20\% spécifiquement aux PMA. Des mesures sont énumérées pour rendre cette aide plus efficace (harmonisation des procédures, assouplissement des modalités de décaissement et d'acheminement de l'APD, abandon de l'aide liée) ;

- La même année, Jacques Chirac s'engage à porter le niveau de I'APD française à 0,5\% du PIB en 2007 et à $0,7 \%$ du PIB en 2012;

- Trois ans plus tard, la conférence des Nations Unies de juin 2005 dresse un premier bilan des engagements de Monterrey. La part du PIB consacrée à I'APD a certes progressé (de 0,24 à $0,33 \%$ pour les pays membres de l'OCDE), mais reste éloignée des objectifs affichés. L'UE propose un nouvel échéancier pour atteindre à terme les $0,7 \%$ et fixe un objectif intermédiaire de $0,56 \%$ en 2010 avant d'atteindre les $0,7 \%$ en 2015. L'UE s'engage également à augmenter I'APD à l'Afrique de $50 \%$ à partir de 2006 ;

- Le mois suivant, le sommet du G8 de Gleneagles (juillet 2005) établit qu'il est nécessaire d'augmenter l'aide de 50 milliards de dollars par an dès 2006, dont 25 milliards pour l'Afrique, pour atteindre les OMD. L'engagement pris à Gleneagles est d'augmenter I'APD de 50 milliards de dollars par an d'ici 2010, dont 25 milliards pour l'Afrique, ce qui correspondrait à un doublement de cette aide pour le continent ;

- Le sommet du G8 de juillet 2008 à Toyako, au Japon, prend acte du fait que I'APD nette des pays de l'OCDE a diminué de 2005 à 2007, et que l'aide à l'Afrique n'a pas décollé, plafonnant à 26 milliards de dollars. Le sommet renouvelle l'engagement de doubler l'aide à l'Afrique d'ici 2010, pour la faire passer de 25 à 50 milliards de dollars par an ;

- Suite au sommet de la FAO à Rome en juin 2008, 22 milliards de dollars sont promis pour apporter des réponses à la crise alimentaire et investir durablement dans l'agriculture ;

- Le sommet du G8 à L'Aquila, en juillet 2009, prend acte du fait que les 22 milliards n'ont pas été versés ni ne risquent de l'être, et réaffirme le principe de coordination des actions et de soutien à la mise en œuvre des politiques nationales. La promesse du sommet, légèrement revue à la baisse, est d'engager 20 milliards de dollars sur trois ans en faveur de la sécurité alimentaire des pays pauvres.

piètres contributeurs européens, I'Italie et la Grèce $(0,20 \%)$;

- le constat de promesses non tenues est le même si l'on se réfère aux montants annoncés lors des différents sommets. La promesse faite, à Gleneagles en 2005, d'augmenter I'APD de 50 milliards de dollars d'ici 2010, dont 25 milliards pour I'Afrique, est très mal partie, puisque l'APD nette des

pays de l'OCDE est passée de 107 milliards de dollars en 2005 à 104 milliards en 2006 et à 103 milliards en 2007. Le décalage entre promesses et réalité est également flagrant dans le cas de l'Afrique, continent pour lequel I'aide devait être doublée pour passer de 25 milliards de dollars de 2005 à 50 milliards en 2010 : en 2008 , on en était péniblement à 26 milliards !

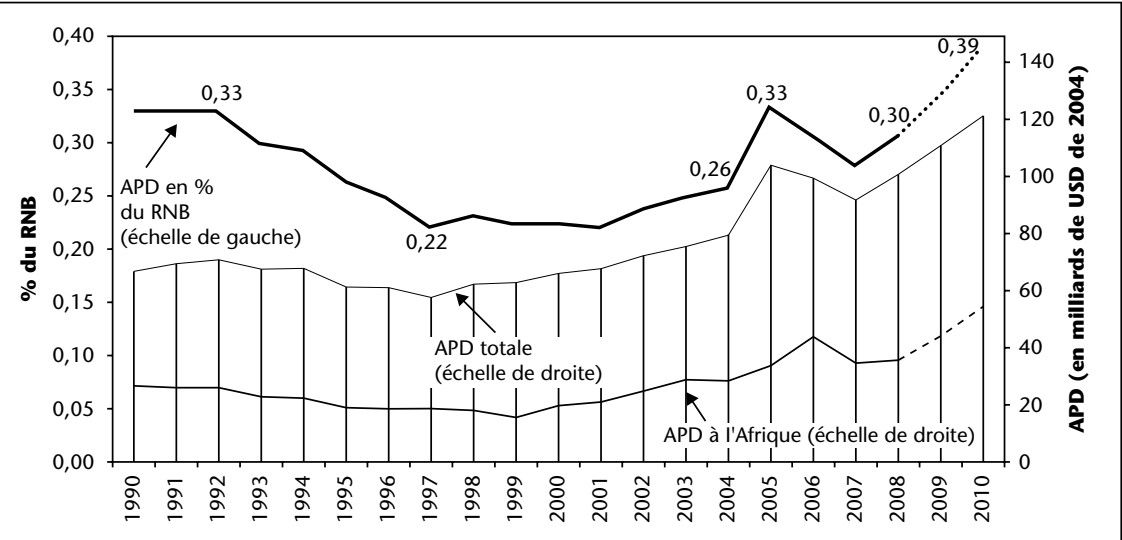

Figure 1. Aide publique au développement (APD) nette des membres du comité d'aide au développement (CAD) de 1990 à 2008 et simulations du CAD pour la période 2009 à 2010, source OCDE, mars 2009.
- les promesses suite à la crise alimentaire de 2008 suivent à l'évidence le même chemin : sur les 22 milliards de dollars promis lors de la conférence de la FAO en juin 2008, seuls 2,3 milliards ont été effectivement décaissés au début 2009. Ce qui n'empêche aucunement de lancer de nouvelles promesses au sommet du G8 à L'Aquila, en juillet 2009, de mobiliser 20 milliards de dollars sur trois ans pour renforcer la sécurité alimentaire des pays les plus pauvres. Qui pourrait y croire?

\section{L'APD mesure mal le financement du développement}

Severino et Ray (2009) soulignent que les données de l'APD mesurent très mal le financement du développement : d'une part, en prenant en compte toute une série de flux financiers non directement liés aux actions de développement ; d'autre part, en négligeant les sources de financement en dehors des 22 pays membres de I'OCDE. Ces sources ont pourtant pris une importance croissante au cours des dix dernières années : I'aide au développement financée par les pays émergents est en forte augmentation. La Chine est ainsi devenue en quelques années le principal financeur des infrastructures en Afrique subsaharienne. Les pays pétroliers du monde arabe ont des politiques d'aide très actives sans que ces dernières soient comptabilisées dans I'APD. Raj (2008) estime à 50 milliards de dollars la contribution annuelle de la solidarité privée (ONG, fondations, etc.) aux actions de développement.

À l'inverse, une bonne part des flux comptabilisés dans l'APD ne finance pas directement des actions de développement. Raj (2008) estime en effet qu'environ $60 \%$ de l'APD correspond à des remises de dette, de la coopération technique, de l'aide alimentaire et des aides humanitaires ou d'urgence, toutes actions dont la portée ne saurait être minimisée, mais qui ne peuvent être considérées comme un apport d'argent frais destiné à financer le développement des pays récipiendaires. L'aide d'urgence en direction des zones de catastrophes naturelles ou de conflits a fortement augmenté depuis dix ans; ce type de dépenses est certes important en termes de soulagement de la détresse humaine, mais son lien avec le développement économique et social des pays récipiendaires est malgré tout assez ténu.

Les annulations de dette constituent sans aucun doute le facteur principal d'évolution des montants d'APD au cours des années 2000. La hausse de l'APD, observée de 2000 à 2005, est pour une large part liée à des annulations de dette qui ont été massives dans la première moitié de la décennie. Pour les mêmes raisons mais en sens opposé, la baisse des annu- 


\section{Encadré 1 \\ Quelques caractéristiques de I'APD agricole}

- La part de l'agriculture dans l'APD a fortement chuté au cours des 30 dernières années : elle est passée de $17 \%$ en 1980 à $8 \%$ à la fin des années 1990 et à 3,8\% en 2006 ;

- les financements multilatéraux à l'agriculture ont été beaucoup plus fortement réduits que les financements bilatéraux. Ils sont passés de 3,4 milliards de dollars en 1980 à 0,5 milliard au milieu des années 2000 (une chute de $85 \%$ ), alors que les financements bilatéraux ne sont baissés que de 2,8 à 1,7 milliard sur la même période (baisse de $39 \%$ ). Du coup, les financements bilatéraux sont devenus très nettement majoritaires dans l'APD agricole, ce qui n'était pas le cas auparavant ;

- l'aide à l'agriculture s'est redéployée depuis les années 1980 :

- le soutien direct à l'agriculture, notamment en matière d'intrants, de services agricoles (incluant le financement), de recherche et de vulgarisation, a nettement chuté ; cela reflète le changement de perception quant au rôle du secteur public dans la fourniture d'intrants et de services agricoles ;

- les projets d'appui à une zone géographique ou à une filière spécifique ont été partiellement remplacés par un appui institutionnel aux politiques agricoles ;

- I'appui à la gestion des ressources naturelles, à l'agroforesterie et à la pêche a crû en termes relatifs;

- le désengagement des bailleurs vis-à-vis de l'agriculture s'est opéré en faveur des infrastructures et services sociaux dont la part dans l'APD est passée de 9 à $33 \%$;

- I'Asie a été la région la plus touchée par la chute des financements à l'agriculture. L'APD agricole a chuté de $83 \%$ en Asie centrale et du Sud, alors qu'elle a été divisée par deux en Afrique subsaharienne. Cette région est devenue de ce fait la principale destinatrice de I'aide agricole (37\%), devant I'Asie du Sud-Est (25\%), I'Asie centrale et du Sud (23\%) et I'Amérique latine (11\%). Dans le cas de la CEDEAO (région d'Afrique de l'Ouest), la part de I'APD allouée au secteur agricole a été de 4,7\% en moyenne sur la période 2001-2007, soit légèrement plus que les $3,8 \%$ toutes régions confondues.

Sources : CAD/OCDE, 2009 ; DFID, 2004

lations de dette, à partir de 2006, a entraîné de manière comptable une baisse du montant total de l'APD en 2006 et en 2007. Les annulations de dette sont pour une large part un assainissement comptable de créances qui n'auraient pas toujours pu être remboursées par les pays débiteurs plutôt qu'un transfert effectif de nouvelles ressources vers le pays, et leur prise en compte dans I'APD par le CAD tend à surestimer l'ampleur des flux financiers nets ${ }^{1}$. II arrive même que la remise de dette corresponde à des activités qui n'étaient aucunement liées au développement: la dette de I'Irak a largement servi à financer l'effort de guerre contre I'Iran (achat de matériel militaire sophistiqué) et sur les 6,5 milliards de dollars comptabilisés comme APD pour I'Irak en 2006, environ la moitié correspond à une remise de dette.

\section{"Culture » du non-respect des promesses?}

Le non-respect des engagements pris à titre individuel ou collectif par la communauté des

\footnotetext{
${ }^{1}$ Concernant l'impact de l'annulation de dette sur le montant de l'APD, voir les notes de travail de Coordination Sud.
}

bailleurs est un problème structurel qui dépasse largement le cadre de la crise actuelle. Les échéances ne sont jamais tenues et sont sans cesse repoussées, avec l'idée que l'avenir sera plus porteur à moyen terme, et que les engagements seront alors plus faciles à tenir. Il est possible de parler à cet égard de culture de non-respect des engagements, qui sont d'autant plus faciles à prendre qu'il n'y a aucune sanction s'ils ne sont pas tenus. II y a une profonde asymétrie dans la relation entre bailleurs et pays récipiendaires: les bailleurs sont en mesure d'appliquer des mesures de rétorsion si les pays récipiendaires ne respectent pas les termes du contrat; la réciproque n'est pas vérifiée, et les bailleurs n'ont strictement rien à craindre s'ils ne respectent pas leurs engagements. Cet état de fait a peu de chances de changer dans un proche avenir, et l'on pourra continuer à dire que les promesses n'engagent que ceux qui y croient !

\section{APD agricole en net retrait}

Caractériser précisément la part de I'APD qui revient au secteur agricole pose divers problèmes d'ordre méthodologique. Les statistiques du CAD/OCDE ne prennent en compte que les actions spécifiquement dirigées au secteur agricole, à l'exclusion de l'aide agricole incluse dans des programmes multisectoriels. De même, l'aide agricole transitant par les ONG n'est prise en compte que si elle est référencée comme telle, ce qui n'est pas souvent le cas.

Les préventions méthodologiques ne sauraient, toutefois, occulter la tendance lourde d'un fort désengagement de l'APD vis-à-vis du secteur agricole, comme cela apparaît dans l'encadré 1.

Ce désengagement de I'APD vis-à-vis du secteur agricole obéit à une tendance lourde, à savoir le changement des objectifs prioritaires de l'aide : ceux-ci sont passés d'un soutien à la croissance des «pays frères » dans le cadre de la guerre froide à la promotion des meilleures conditions de vie des populations, incarnées dans les objectifs du millénaire pour le développement (OMD), après la chute du mur de Berlin (Severino et Ray, 2009). De nombreux bailleurs, et tout particulièrement les bailleurs multilatéraux, ont ainsi donné la priorité aux dépenses sociales dans les domaines de la santé et de l'éducation ; les programmes d'allégement de la dette ont également détourné les ressources disponibles des secteurs productifs tels que l'agriculture. À cette tendance lourde se sont ajoutées les difficultés inhérentes au secteur agricole : de par ses caractéristiques propres (activité productive fortement aléatoire, grande volatilité des prix, multiplicité des acteurs), l'agriculture est un secteur complexe dans lequel le taux d'échec des projets est élevé. Ces divers éléments ont incité les responsables d'agences de développement à se détourner du secteur agricole, et à en privilégier d'autres dans lesquels le taux de réussite est supérieur, les coûts de transaction de mise en œuvre et de suivi des projets plus restreints, et les conditions de décaissement plus faciles. La tendance naturelle des bailleurs à se détourner du secteur agricole s'est trouvée confortée par la faible demande de soutien à l'agriculture formulée par les pays récipiendaires. Nombre de ceux-ci ont en effet délaissé le secteur agricole de leur propre agenda, avec pour conséquence une baisse substantielle de la part de leur budget national consacré à l'agriculture. Cette part, fréquemment supérieure à $15 \%$ dans les années 1980, a fortement chuté depuis lors pour ne plus représenter que quelques pour-cents au début des années 2000. L'engagement des chefs d'État africains, pris à Maputo en 2003, de consacrer $10 \%$ de leur budget à l'agriculture n'a pas véritablement été suivi d'effets : cette part reste le plus souvent comprise actuellement entre 4 et $8 \%$.

Le déclic d'un regain d'intérêt pour la question agricole semblait s'être produit entre la fin 2007 et l'été 2008 au niveau de la communauté internationale. Les premiers signes 
s'étaient manifestés avec la publication du rapport sur le développement dans le monde 2008 de la Banque Mondiale, consacré à l'agriculture pour la première fois depuis 25 ans. Ce rapport soulignait, études économétriques à l'appui, qu'il était stratégique de miser sur le secteur agricole dans la mesure où un point de croissance dans ce secteur avait beaucoup plus d'impact sur la réduction de la pauvreté que cette même croissance dans un autre secteur d'activité. La crise alimentaire de 2008 a également contribué à faire remonter l'agriculture sur le devant de la scène des débats internationaux : une dépendance trop forte vis-à-vis des marchés internationaux peut se révéler catastrophique lors d'envolées des cours mondiaux, et il est donc souhaitable de couvrir une partie substantielle des besoins alimentaires par la production nationale ou régionale.

Ce regain d'intérêt pour l'agriculture n'a toutefois pas duré. Les promesses d'aide formulées lors du sommet de la FAO de juin 2008 n'ont pas été tenues. Elles ont été reformulées à la baisse lors du sommet du G8 à L'Aquila en juillet 2009 (20 milliards de dollars sur trois ans), et celles-ci ne seront très probablement pas tenues non plus. Le récent sommet de la FAO de novembre 2009 est révélateur du climat général de désengagement de la communauté internationale. Silvio Berlusconi a été le seul chef d'État à participer au sommet, alors que la rencontre précédente de juin 2008 avait donné lieu à une mobilisation impressionnante. De plus, le récent sommet ne s'est conclu que sur des déclarations de principe, sans même aucun engagement chiffré.

\section{À la recherche de nouveaux partenariats}

Le débat sur l'APD ne saurait se limiter aux seuls volumes promis et/ou effectivement décaissés. II porte également sur son efficacité, en lien avec les modalités de mise en œuvre. Des voix se sont élevées récemment pour dénoncer non seulement l'inefficacité de l'aide, mais aussi dans certains cas sa nocivité. Cette critique n'est pas nouvelle, mais elle a pris depuis quelque temps une ampleur qu'elle $n^{\prime}$ avait pas jusqu'alors. Glennie (2008) souligne certains effets pervers de l'aide (création d'une situation de dépendance, fragilisation des institutions locales, renforcement de pouvoirs non démocratiques, imposition de politiques qui ont appauvri certaines parties de la population, etc.) et milite pour une révision des modalités $d$ 'intervention de l'aide avant de penser à en augmenter les volumes. II reprend ainsi les arguments développés par Killick (2007) qui plaide pour la primauté de la qualité de l'aide par rapport à la quantité, en insistant sur deux aspects :

- une efficacité marginale décroissante de I'aide plus les volumes augmentent ;

- une faible capacité d'absorption de l'aide : plus les institutions du pays récipiendaire sont fragiles, moins elles sont en mesure d'absorber I'aide sans entraîner d'effets pervers.

D'autres critiques de l'aide sont plus radicales et considèrent celle-ci comme systématiquement nuisible et devant être purement et simplement éliminée. C'est le cas de Moyo (2009), pour qui toute aide est génératrice de distorsions graves, quelles qu'en soient les modalités. II faut donc la supprimer et laisser les États se développer tous seuls, sans intervention extérieure.

Dans les débats récents, deux effets pervers de I'aide sont tout particulièrement évoqués :

- la fragilisation des instances démocratiques nationales;

- la confiscation de la souveraineté nationale au profit d'un pilotage stratégique exercé de l'extérieur. Ils sont de portée générale, mais s'observent clairement dans le secteur agricole.

\section{Fragilisation des instances démocratiques nationales}

Malgré l'objectif affiché de l'APD de renforcer les institutions des pays récipiendaires de l'aide, les résultats en la matière ont rarement été au rendez-vous ; ils ont même été souvent contraires aux effets attendus. Au lieu de renforcer les capacités institutionnelles des gouvernements, la multiplication d'initiatives faiblement coordonnées a contribué à un affaiblissement des ressources locales, notamment au niveau des instances publiques en charge du secteur agricole.

La mise en œuvre des programmes d'ajustement structurel (PAS), au cours des années 1980 et 1990, a conduit de nombreux gouvernements à réduire les moyens financiers et humains de leurs administrations. Les PAS ont ainsi provoqué le départ de nombreux fonctionnaires; ceux qui restent sont le plus souvent âgés (la pyramide des âges de la plupart des ministères de l'Agriculture africains montre une surreprésentation des 50-60 ans par rapport aux 20-30 ans et 30-40 ans), et le faible niveau des rémunérations les conduit à chercher des compléments de revenu en dehors de leur activité principale.

Cet affaiblissement des administrations nationales s'est poursuivi et renforcé pendant la dernière décennie du fait de la propension des bailleurs à créer, pour mettre en œuvre leurs projets, des unités de gestion parallèles qui concurrencent directement les services techniques en place, au lieu de les renforcer. Fréquemment méfiants à l'égard d'administra- tions publiques considérées comme inefficaces, les bailleurs de fonds ont préféré, dans leur grande majorité, contourner les structures nationales et constituer des unités parallèles qui leur sont dévouées. L'attitude de défiance des bailleurs de fonds vis-à-vis des administrations comporte un aspect inéluctable de prophétie autoréalisatrice : les choix opérés par de nombreux bailleurs au nom de l'inefficacité de l'État contribuent en effet à affaiblir un peu plus des administrations déjà exsangues, et à les rendre de fait plus inefficaces. Les conséquences négatives de la création de structures ad hoc non directement liées aux administrations se manifestent à plusieurs niveaux (Ribier et Le Coq, 2007) :

- en premier lieu, les ressources humaines du gouvernement sont accaparées par la gestion à court terme des projets, le suivi des procédures et les missions d'évaluation propres à chaque bailleur. II est à signaler à cet égard que les coûts de transactions de l'aide à charge des pays récipiendaires sont d'autant plus élevés que le niveau de coordination est faible entre bailleurs. Cet état de fait diminue d'autant la capacité des équipes nationales à élaborer et à mettre en œuvre des politiques publiques ;

- par ailleurs, les équipes gouvernementales supposées être en charge des dossiers et garantes de la cohérence de la stratégie nationale se trouvent dessaisies et déresponsabilisées; elles disposent d'encore moins de moyens qu'auparavant et sont, de plus, largement décrédibilisées aux yeux des différents types d'acteurs nationaux et internationaux ;

- enfin, le développement d'opportunités d'emplois beaucoup mieux rémunérés que dans la fonction publique ${ }^{2}$ provoque le départ de l'administration d'une partie des cadres les mieux formés et les plus à même de saisir ces opportunités. Les différentiels de salaire, d'équipement et de conditions de travail représentent une force $d^{\prime}$ attraction souvent irrésistible pour le personnel des administrations, qui évolue donc de projet en projet ou est directement embauché par les partenaires techniques et financiers (PTF). De manière contradictoire, les PTF qui se plaignent de la faible capacité des administrations, voire ceux qui financent des projets de renforcement de capacités, sont parfois aussi ceux qui recrutent des experts de l'administration pour leur représentation locale (Crola, 2009).

\footnotetext{
${ }^{2}$ À titre d'exemple, à Madagascar, le salaire des responsables nationaux de développement dans une ONG est fréquemment de l'ordre de quatre fois ce que ce même responsable aurait pu obtenir au ministère de l'Agriculture (MAEP).
} 


\section{Confiscation de la souveraineté nationale}

L'un des objectifs les plus couramment affichés de l'APD est I'amélioration de la gouvernance et le renforcement de la capacité des administrations à élaborer des stratégies de développement et à mettre en œuvre les politiques publiques correspondantes. C'est d'ailleurs le premier principe de la Déclaration de Paris, renforcement de l'ownership, terme anglais dont la traduction française « appropriation » est très équivoque. On ne s'approprie que ce qui n'était initialement pas à soi ; il serait donc plus indiqué de parler de "maîtrise des politiques publiques» comme le font les Canadiens, de «souveraineté politique» ou encore $\mathrm{d}^{\prime}$ « auto-détermination» pour reprendre un terme historiquement daté.

Le caractère équivoque du terme appropriation est d'ailleurs symptomatique de la position de certains bailleurs qui le comprennent comme l'engagement des gouvernements bénéficiaires en faveur des politiques et des réformes, sans considérer la façon dont ces dernières ont été décidées. De Renzio et al. (2008) proposent la définition suivante : "I'appropriation est le degré de contrôle que les gouvernements bénéficiaires de l'aide sont capables d'exercer sur le choix, la formulation et la mise en œuvre des politiques publiques et des réformes dans leur pays ». II existe bien un décalage persistant chez nombre de bailleurs entre la position affichée de soutien au leadership du pays récipiendaire et la pratique de promouvoir ses propres vues, dès lors que le gouvernement en question exprime des préférences différentes.

Ces considérations sémantiques étant établies, force est de constater que les résultats de l'aide sur la promotion de leadership national sont des plus mitigés, voire bien souvent négatifs : I'APD ne contribue que trop rarement à redonner de la souveraineté ou une marge de manœuvre supplémentaire aux gouvernements récipiendaires. Ce constat général recouvre différentes réalités :

- certains gouvernements utilisent l'aide pour se maintenir au pouvoir, pour tenir leurs promesses électorales, mais aussi pour asseoir une légitimité qu'ils ont du mal à obtenir par ailleurs. Ils sont de ce fait réticents à élaborer une stratégie de développement ou des positions politiques fortes qui pourraient s'écarter des préférences des bailleurs de fonds, de peur de voir le volume d'aide diminuer, et ainsi entamer leur base politique ou entraîner des risques de perdre les prochaines élections ;

- d'une manière plus générale, la faible légitimité politique de certains gouvernements récipiendaires combinée à leur dépendance vis-à-vis de l'aide internationale crée des incita- tions fortes à rester dans une position subordonnée vis-à-vis des bailleurs de fonds ;

- les conditions de négociation permanente, l'enchevêtrement des agences d'aide internationale au sein même des institutions nationales et la fragmentation de l'aide constituent des obstacles à la remise en cause de cette subordination. En conséquence de quoi les dirigeants politiques finissent par accepter les propositions des bailleurs de guerre lasse, même si elles ne correspondent pas à leurs priorités ; - de nombreux bailleurs ont une idée très prescriptive du type de politiques qu'ils veulent promouvoir dans les pays aidés, ce qui tend à laisser peu de place aux cadres nationaux pour élaborer eux-mêmes des politiques de développement et pour mettre en place les programmations et plans opérationnels correspondants. Par perte d'habitude, faible incitation ou incapacité, les autorités publiques sont enclines à « acheter » sans condition les solutions proposées par les institutions financières internationales et autres PTF plutôt qu'à écouter les propositions de certains cadres expérimentés et volontaires de leur administration (Bergamaschi et al., 2007).

\section{Appropriation et conditionnalités : le couple infernal?}

La Déclaration de Paris de mars 2005, signée conjointement par bailleurs et pays bénéficiaires, prend acte des difficultés rencontrées par l'aide, et met en avant cinq principes dont l'application devrait permettre une amélioration de son efficacité : appropriation, alignement, harmonisation, gestion par les résultats et responsabilité mutuelle. La Déclaration de Paris (2005) propose également une évolution des modalités de l'aide, dans le sens d'une réduction de l'aide projet au profit de l'aide budgétaire, dont les effets attendus sont le renforcement de l'État, la réduction des coûts de transaction, une meilleure coordination entre bailleurs et une plus grande prévisibilité de I'aide (Solanet et Chellali, 2009).

Si l'aide budgétaire paraît effectivement répondre dans son esprit au souhait de promouvoir la souveraineté politique des pays bénéficiaires, les conditions de sa mise en œuvre opérationnelle se sont toutefois avérées très problématiques. La communauté du développement se trouve confrontée à un dilemme : bailleurs de fonds et pays récipiendaires s'accordent sur un schéma théorique d'orientation stratégique, mais ils ne parviennent pas à trouver les modalités pour le rendre effectif (Samuel, 2009). L'octroi de l'aide budgétaire est bien souvent conditionné à l'obtention de résultats qui sont évalués à l'aide d'indicateurs de différentes natures. Plus que les anciennes modalités de I'APD, I'aide budgétaire accorde un rôle pré- pondérant à la gestion orientée sur les résultats et à la production d'indicateurs chiffrés destinés à évaluer si les objectifs assignés ont été atteints. Le choix des objectifs et la mesure des indicateurs de suivi posent divers problèmes d'ordre méthodologique :

- le premier constat est que la programmation par objectifs est compliquée, car il est difficile $d^{\prime}$ identifier des objectifs non ambigus, pas trop ambitieux, aisés à atteindre ;

- les indicateurs de résultats que les gouvernements doivent renseigner sont le plus souvent très nombreux. À titre d'exemple, au Mali, la matrice des indicateurs du cadre d'évaluation conjointe (CEC) ne contient pas moins de 51 indicateurs dans 16 domaines différents ;

- outre leur nombre important, les indicateurs sont bien souvent hétérogènes entre eux, et leur superposition pose un problème de cohérence : la mise bout à bout d'informations relatives au taux de croissance, au niveau du stock de sécurité en céréales, au niveau d'infection des cours d'eau, ne permet pas d'avoir une vision globale de l'amélioration de la situation du pays;

- un autre problème vient de la difficulté à mesurer nombre d'indicateurs; certains sont par nature difficilement mesurables; d'autres requièrent des données qu'il est malaisé d'obtenir, ou qui demandent du temps et des moyens; le problème est d'autant plus aigu quand il s'agit d'indicateurs périodiques tels que des indicateurs annuels. Les sources $d^{\prime}$ information qui renseignent les indicateurs sont bien souvent multiples et reposent sur des méthodologies différentes: les données produites ne sont alors pas fiables ;

- les indicateurs retenus sont souvent trop réducteurs pour apprécier véritablement les résultats. En outre, ils ne sont pas toujours adaptés au suivi de la « performance » des gouvernements : I'amélioration de nombre d'entre eux peut en effet ne pas être du ressort direct du gouvernement.

Le rôle prépondérant du pilotage par les indicateurs est lourd de conséquences sur les modalités de gestion de l'aide, sur les relations entre bailleurs et récipiendaires, et par voie de conséquence sur l'efficacité de l'aide :

- il y a un fort contraste entre d'une part l'aspect minutieux, voire pointilleux du suivi des multiples indicateurs, secteur par secteur, et, d'autre part, l'absence de vision claire de la part des bailleurs sur les évolutions souhaitables de I'APD ;

- la focalisation sur les résultats budgétaires fait perdre de vue les priorités du développement et l'amélioration de la situation des populations (à titre d'exemple, au Mali, I'accent a été mis sur l'atteinte d'objectifs budgétaires tels que la privatisation de la compagnie malienne pour le développement des textiles, CMDT, sans se 
soucier des conséquences sur les conditions de vie des producteurs de coton qui ont vu se dégrader leurs revenus) ;

- au-delà des seules considérations budgétaires, la forte polarisation sur des indicateurs quantitatifs ne laisse pas assez l'opportunité de travailler sur les processus ;

- par ailleurs, l'espoir d'une réduction de la volatilité de l'aide est bien souvent battu en brèche par la réalité. La crédibilité du système de suivi par indicateurs impose de suspendre les décaissements en cas de résultats décevants, ce qui entraîne alors des discontinuités budgétaires et des ajustements fiscaux douloureux (Rogerson, 2005).

Au total, on se trouve bien confrontés au paradoxe suivant : les réformes récentes de l'aide (les cadres stratégiques de lutte contre la pauvreté, les approches sectorielles, le recours croissant à l'aide budgétaire globale) qui visaient à résoudre les problèmes des systèmes de gestion de l'aide fragmentés ou parallèles, et par là même à redonner du leadership aux pays bénéficiaires, ont bien souvent eu des effets inverses en entraînant une plus grande dépendance de ces derniers ; les nouvelles modalités ont en fait accru l'intrusion des bailleurs dans les arènes de la prise de décision et ont créé une confusion croissante entre les institutions nationales et des initiatives ou structures promues et poussées par les bailleurs de fonds (de Renzio et al., 2008). Les logiques d'intervention des bailleurs de fonds, contraintes par des procédures rigides et une vision courttermiste et restrictive de l'État comme gestionnaire de l'aide, conduisent à un renforcement des capacités de gestion de l'aide, mais pas des capacités à mettre en œuvre des politiques publiques propres: les compétences technocratiques de gestion sont survalorisées aux dépens des compétences réelles en politiques de développement.

\section{Conclusion}

L'APD a fortement évolué depuis la période des indépendances. Le contexte géopolitique mondial a subi une transformation profonde, passant d'un monde bipolaire où chaque camp soutenait ses « pays amis » à un paysage post-guerre froide beaucoup plus éclaté dans lequel des liens d'interdépendance planétaire se sont affirmés (pandémies, migrations, changement climatique, etc.). L'émergence de ces nouveaux enjeux a contribué à orienter l'aide, dont les objectifs se sont diversifiés. Cette évolution appelle à une plus grande responsabilisation des pays bénéficiaires, pour qu'ils apportent leur contribution à la résolution de la problématique mondialisée.

Prenant en compte la montée des nouveaux enjeux de l'aide, les regards se font de plus en plus critiques. Ils sont toutefois l'expression de sensibilités différentes. Certains, plutôt favorables à l'aide, regrettent le manque d'ambition des pays riches qui n'engagent pas les moyens à la hauteur des enjeux; ils constatent avec amertume la culture de non-respect des promesses formulées au cours des différents sommets internationaux. D'autres, plus critiques, plaident davantage pour une réforme des modalités de l'aide que pour un accroissement de ses volumes; ils soulignent la difficulté à atteindre dans les faits les objectifs affichés dans la Déclaration de Paris, la restauration du leadership des pays bénéficiaires en premier lieu. D'autres enfin expriment une défiance totale vis-à-vis de l'aide, quelles qu'en soient les modalités. En faisant remarquer que dans l'aide au développement, l'important n'est pas l'aide mais le développement, ils dénoncent l'inversion des priorités, qui se traduit par une focalisation trop exclusive sur des solutions venant de l'extérieur, alors que les ressorts du développement sont $d$ 'abord internes aux pays concernés. D'autres voies que l'APD peuvent se révéler plus efficaces pour le développement : remise à plat au niveau international des politiques migratoires, lutte contre les paradis fiscaux, meilleur partage de la rente des matières premières.

Quoi qu'il en soit, il est clair que l'APD est en profonde mutation par rapport à ce qu'elle était encore au début des années 2000. La Déclaration de Paris a initié un nouveau mode de partenariat en donnant plus la parole aux pays bénéficiaires. La déception liée aux premiers résultats de l'application des principes de Paris montre que le dialogue politique initié n'est pas encore suffisant et qu'il doit être approfondi à l'avenir.

\section{RÉFÉRENCES}

Bergamaschi I, Diabaté A, Paul E. L'agenda de Paris pour l'efficacité de l'aide. Défis de l'« appropriation » et impact des nouvelles modalités de l'aide au Mali. Afrique contemporaine 2007; $n^{\circ} 3 / 4$.
CAD/OCDE. Données statistiques de I'APD, 1990-2009.

Crola JD. Aide à l'agriculture : des promesses aux réalités de terrain. L'état de la coordination des interventions dans trois pays d'Afrique de l'Ouest. Rapport de recherche ? Oxfam France - Agir ici, 2009; 56 p.

Déclaration de Paris sur l'efficacité de l'aide au développement, 2005.

De Renzio P, Whitfield L, Bergamaschi I. Réformer les pratiques de l'APD : comment les bailleurs de fonds peuvent comprendre l'appropriation et la soutenir. Note d'analyse. Global Economic Governance Programme, 2008.

DFID. Official development assistance to agriculture. DFID working paper, $2004 ; 27$ p.

Gabas JJ. Les pays émergents et la coopération internationale. In: Jaffrelot (dir.) L'enjeu mondial. Les pays émergents. Paris : Presses de Sciences Po 2008 : 221-34.

Glennie J. The trouble with aid: why less could mean more for Africa. Zed Books $2008 ; 175$ p.

Killick T, Foster M. The macroeconomics of doubling aid to Africa and the centrality of the supply side. Development Policy Review 2007 ; 25 : 167-92.

Moyo D. The dead aid, 2009.

Pouilles-Duplaix A. Réponse opérationnelle internationale à la crise des prix alimentaires en 2008. Contribution aux «Rencontres publiques du CSAAD », 28 janvier 2009.

Raj MD. The California consensus: can private aid end global poverty? Survival $2008 ; 50\left(n^{\circ} 4\right)$.

Ribier V, Le Coq JF. Renforcer les politiques publiques agricoles en Afrique de l'Ouest et du Centre : pourquoi et comment? Notes et études économiques du ministère de l'Agriculture et de la Pêche 2007; 28 : 45-73.

Rogerson A. Aid harmonisation and alignment: bridging the gap between reality and the Paris reform agenda. Development Policy Review 2005; 23 : 531-52.

Samuel B. Le cadre stratégique de lutte contre la pauvreté et les trajectoires de la planification au Burkina Faso. Sociétés politiques comparées, $n^{00} 16$, CERI Sciences Po $76 \mathrm{p}$.

Severino JM, Ray O. The end of ODA: death and rebirth of o global public policy. Working Paper $n^{\circ} 167,2009$. Center for Global Development, 28 p.

Solanet $\mathrm{G}$, Chellali K. L'aide budgétaire en question: quelle efficacité, pour qui? Groupe de travail sur I'efficacité de l'aide Gret-Coordination Sud. Collection Débats et Controverses 2009, n² 2, Éditions du Gret. 